\title{
Unravelling the evolutionary origins of $X$ chromosome inactivation in mammals: insights from marsupials and monotremes
}

\author{
Janine E. Deakin • Julie Chaumeil • \\ Timothy A. Hore $\cdot$ Jennifer A. Marshall Graves
}

(C) Springer Science + Business Media B.V. 2009

\begin{abstract}
Determining the evolutionary origin of $\mathrm{X}$ inactivation mechanisms in mammals requires knowledge of features of $\mathrm{X}$ inactivation across all three major mammal lineages; monotremes, marsupials and eutherians. In the past, research into $\mathrm{X}$ inactivation in marsupials and monotremes lagged far behind the major advances made in understanding the mechanisms of $\mathrm{X}$ inactivation in human and mouse. Fragmentary knowledge of the genic content and sequence of marsupial and monotreme $\mathrm{X}$ chromosomes has been alleviated by the recent release of genome sequences for two marsupials and one monotreme. This has lead to a number of important findings, among which is the absence of XIST in marsupials and monotremes, and the surprising finding that X-borne genes in platypus are subject to stochastic transcriptional inhibition rather than whole chromosome inactivation. Availability of sequence data, and new techniques for studying expression and
\end{abstract}

Responsible Editor: Edith Heard.

J. E. Deakin $(\bowtie) \cdot$ T. A. Hore $\cdot$ J. A. Marshall Graves

Comparative Genomics Group, School of Biology,

College of Medicine, Biology and Environment,

The Australian National University,

Canberra ACT 0200, Australia

e-mail: Janine.Deakin@anu.edu.au

J. Chaumeil

Pathology Department,

New York University School of Medicine,

New York, NY 10016, USA chromatin modification, now make rapid advance possible.

Keywords X chromosome inactivation . dosage compensation $\cdot$ marsupial $\cdot$ monotreme
Abbreviations
ATR ataxia telangiectasia and $\operatorname{Rad} 3$ related
BRCA1 breast cancer 1
DMRT1 doublesex and mab-3 related transcrip- tion factor 1
G6PD glucose-6-phosphate dehydrogenase
GLA galactosidase, alpha
H3K4me2
$\mathrm{H} 3 \mathrm{~K} 9 \mathrm{ac}$ histone $\mathrm{H} 3$ dimethylation on lysine 4
H3K9me2 histone $\mathrm{H} 3$ acetylation on lysine 9
H3K27me3 histone $\mathrm{H} 3$ dimethylation on lysine 9
$\mathrm{H} 4 \mathrm{Kac}$ histone $\mathrm{H} 3$ trimethylation on lysine 27
HP1 $\beta$
HP1 $\gamma$ histone $\mathrm{H} 4$ acetylation
HPRT1 heterochromatin protein $1 \beta$ heterochromatin protein $1 \gamma$
LINE1 long interspersed nuclear element 1
MHM male hypermethylated
MSCI meiotic sex chromosome inactivation
MYA million years ago
PGK1 phosphoglycerate kinase 1
RNA-FISH ribonuleic acid fluorescent in situ hybridisation
RT-PCR reverse transcriptase polymerase chain reaction 


$\begin{array}{ll}\text { SLC1A1 } & \text { solute carrier family } 1 \text { member } 1 \\ \text { SLC16A2 } & \text { solute carrier family 16, member } 2 \\ \text { SNP } & \text { single nucleotide polymorphism } \\ \text { SNuPE } & \text { single nucleotide primer extension } \\ \text { TSIX } & \text { XIST antisense RNA } \\ \text { XCI } & \text { X chromosome inactivation } \\ \text { XIC } & \text { X inactivation centre } \\ \text { XIST } & \text { X-inactive specific transcript } \\ \text { Xite } & \text { X-inactivation intergenic transcription } \\ & \text { element } \\ \gamma H 2 A X & \text { Phosphorylated histone H2AX }\end{array}$

\section{Introduction}

The $\mathrm{X}$ chromosome of all species of placental mammals ('eutherians'; $e u=$ true, therian=beast) have a suite of about 1100 genes that is virtually identical across species, and whose order is also highly conserved even between human and many other species (rodents are an exception). The $\mathrm{Y}$ chromosome is very much smaller, having lost all but a few of these genes (Graves et al. 2006), leaving genes on the $\mathrm{X}$ present in two doses in XX females and a single dose in $\mathrm{XY}$ males. The chromosome-wide silencing of genes on one $\mathrm{X}$ chromosome in eutherians compensates for this difference in gene dosage. This inactivation of one $\mathrm{X}$ chromosome occurs randomly in cells of the early embryo, and is stable and somatically heritable.

For over 50 years, $\mathrm{X}$ chromosome inactivation and the mechanisms behind it have been extensively studied in humans, mice and common domestic mammals. The $\mathrm{X}$ chromosome in eutherians is inactivated by a complex, multistep process, of which many of the major steps are understood. The initiation of the silencing process during embryogenesis is under the control of the $\mathrm{X}$ inactivation centre (XIC) (Brockdorff et al. 1991; Brown et al. 1991), a master locus encoding several non-coding RNAs, including the XIST (X Inactive Specific Transcript) gene. In each cell of the embryo, interplay of the XICs of each $\mathrm{X}$ chromosome is critical for counting the number of $\mathrm{X}$ chromosomes, choosing the one to inactivate and initiation of silencing (Bacher et al. 2006; Xu et al. 2006; Augui et al. 2007). The XIST transcript then coats the $\mathrm{X}$ chromosome destined for inactivation and initiates formation of a repressive compartment from which RNA polymerase II and other transcription factors are excluded (Chaumeil et al. 2006). The future inactive $\mathrm{X}$ then undergoes a series of chromatin changes through histone modifications and DNA methylation (reviewed by Heard 2005). The condensed chromatin of the inactive $\mathrm{X}$ (visible within the nucleus as a Barr body in humans) undergoes replication later in the cell cycle than the active X.

To begin tracing the evolutionary origins of this complex process, we need to look beyond these familiar eutherian mammals to distantly related members of the class Mammalia. Marsupials (infraclass Metatheria) and monotremes (subclass Prototheria) last shared a common ancestor with eutherian mammals 147 and 166 million years ago (MYA) respectively (Bininda-Emonds et al. 2007). Although eutherians, marsupials and monotremes share the common characteristics of mammals, such as fur and feeding their young milk, each of the three major lineages also differ in many unique attributes. Prominent unique features are their modes of reproduction and, in the case of monotremes, their sex chromosomes, both of which could have implications for their strategies for dosage compensation.

Here we summarize the history of $\mathrm{X}$ inactivation studies in marsupials and monotremes, report on the latest findings and discuss how they can answer some of the questions about the evolution of dosage compensation in mammals.

\section{Marsupials}

Marsupials give birth to immature young and most of their development occurs postnatally while the young are attached to a teat, often within the confines of a pouch. There are 270 species of marsupials, 200 of which live in Australasia and 70 in the Americas (one in North America and 69 in South America). Australian and American marsupials last shared a common ancestor around 70 MYA (Kirsch et al. 1997), so a comparison between American and Australian marsupials is similar in evolutionary terms to the human-mouse comparison which has been so informative for determining the common characteristics of $\mathrm{X}$ inactivation in eutherian mammals.

Early work on $\mathrm{X}$ inactivation in marsupials was carried out on several Australian species (largely kangaroos), and the North American opossum 
(Didelphis virginiana). In more recent years, research has focussed on just two model marsupial species, the tammar wallaby (Macropus eugenii) and the South American opossum (Monodelphis domestica). The genomes for both of these species have been sequenced (Human Genome Sequencing Center at Baylor College of Medicine 2009; Mikkelsen et al. 2007), providing a valuable resource for studies on $\mathrm{X}$ inactivation. The tammar wallaby, a member of the kangaroo family (Macropodidae), is small and easily bred in captivity. Captive animals are derived from two genetically isolated populations (Kangaroo Island in the south and Garden Island in the west of Australia) which differ in many fixed polymorphisms (Zenger et al. 2002). Hybrids between these two populations are an asset for studies where it is important to determine the parental origin of alleles. Opossums (M.domestica) are the marsupial version of the lab mouse or rat, being easily housed and bred in the laboratory, and able to produce many young at once, which is particularly valuable for research into $\mathrm{X}$ inactivation during development.

\section{The Marsupial X Chromosome}

Marsupials have an XX female, XY male sex chromosome system. The marsupial $\mathrm{X}$ shares homology with two-thirds of the eutherian $\mathrm{X}$ chromosome and corresponds to an ancient (150MYA) conserved region of the human $\mathrm{X}$ chromosome (Fig. 1), whereas a recently added region of the human $\mathrm{X}$ is autosomal in marsupials (Graves 1995). Many genes from this added region escape inactivation in humans, presumably reflecting its recent arrival (Carrel and Willard 2005; Johnston et al. 2008). Given the high degree of homology between the marsupial and eutherian X, one might predict that dosage compensation would be similar in these two groups, having arisen from a common mechanism.

There are several clues in the organization of the $\mathrm{X}$ that this may not be the case. Despite conservation of gene content, gene order between marsupials and eutherians has not been retained. Several major rearrangements between the opossum and human $\mathrm{X}$ were revealed during the assembly of the opossum genome (Mikkelsen et al. 2007). Even more surprisingly, gene order is poorly conserved between marsupials, with many rearrangements detected between the opossum and wallaby $\mathrm{X}$ chromosomes
(Deakin et al. 2008b). This contrasts with the eutherian $\mathrm{X}$, which has undergone very little rearrangement since their divergence from marsupials. A suppression of rearrangement on the $\mathrm{X}$ in eutherians was suggested to be a consequence of selection against disruption of the chromosome-wide dosage compensation system (Ohno 1967). Moreover, LINE1 elements are concentrated on the human and mouse $\mathrm{X}$ chromosome, and their concentration correlates to the degree of inactivation, suggesting that they act as 'booster stations' for spread of silencing along the X chromosome (Lyon 1998; Carrel et al. 2006). Sequencing of the opossum $X$ chromosome shows that LINE1 elements have only passively accumulated on the $\mathrm{X}$ and are unlikely to play a critical role in the inactivation process in marsupials (Mikkelsen et al. 2007).

These differences in structure and organization of the marsupial and eutherian $\mathrm{X}$ chromosomes raise the possibility that marsupials have a significantly different dosage compensation mechanism, having independently evolved at least some steps of the $\mathrm{X}$ inactivation mechanism. Does the data on marsupial $\mathrm{X}$-inactivation allow us to recapitulate what steps are in common (and therefore ancient) and what steps are more recent embellishments in one or other lineage? First we will summarize what is known about marsupial $\mathrm{X}$ inactivation, and then consider this information with respect to evolution.

Paternal $\mathrm{X}$ inactivation

As in eutherians, one $\mathrm{X}$ of marsupials was found to be late replicating, leading to the assumption that marsupial $\mathrm{X}$ inactivation is similar to inactivation in human and mouse (Graves 1967). However, biochemical and cytogenetic studies in heterozygous and hybrid animals showed that it is qualitatively different.

The somatic tissues of eutherian mammals are mosaics of cells in which the active $\mathrm{X}$ is the one inherited from the mother or the father. This observation revealed that the decision of which $\mathrm{X}$ to silence is made randomly in cells of the inner cell mass (Lyon 1961). In contrast, early work on isozyme expression showed that marsupial X-inactivation is non-random, with the paternally-derived $X$ being preferentially silenced (Richardson et al. 1971). In hybrid animals with distinguishable maternal and paternal X chromosomes, 
Fig. 1 Dosage compensation features for each of the three major mammalian lineages. The X chromosomes of marsupials and eutherians share homology, whereas the $\mathrm{X}$ chromosomes of monotremes share homology with the chicken $\mathrm{Z}$ chromosome. Putative lineage-specific characteristics and features common to more than one lineage are indicated. (*Paternal XCI in eutherians is observed in the extraembryonic tissues of rodents and cattle)

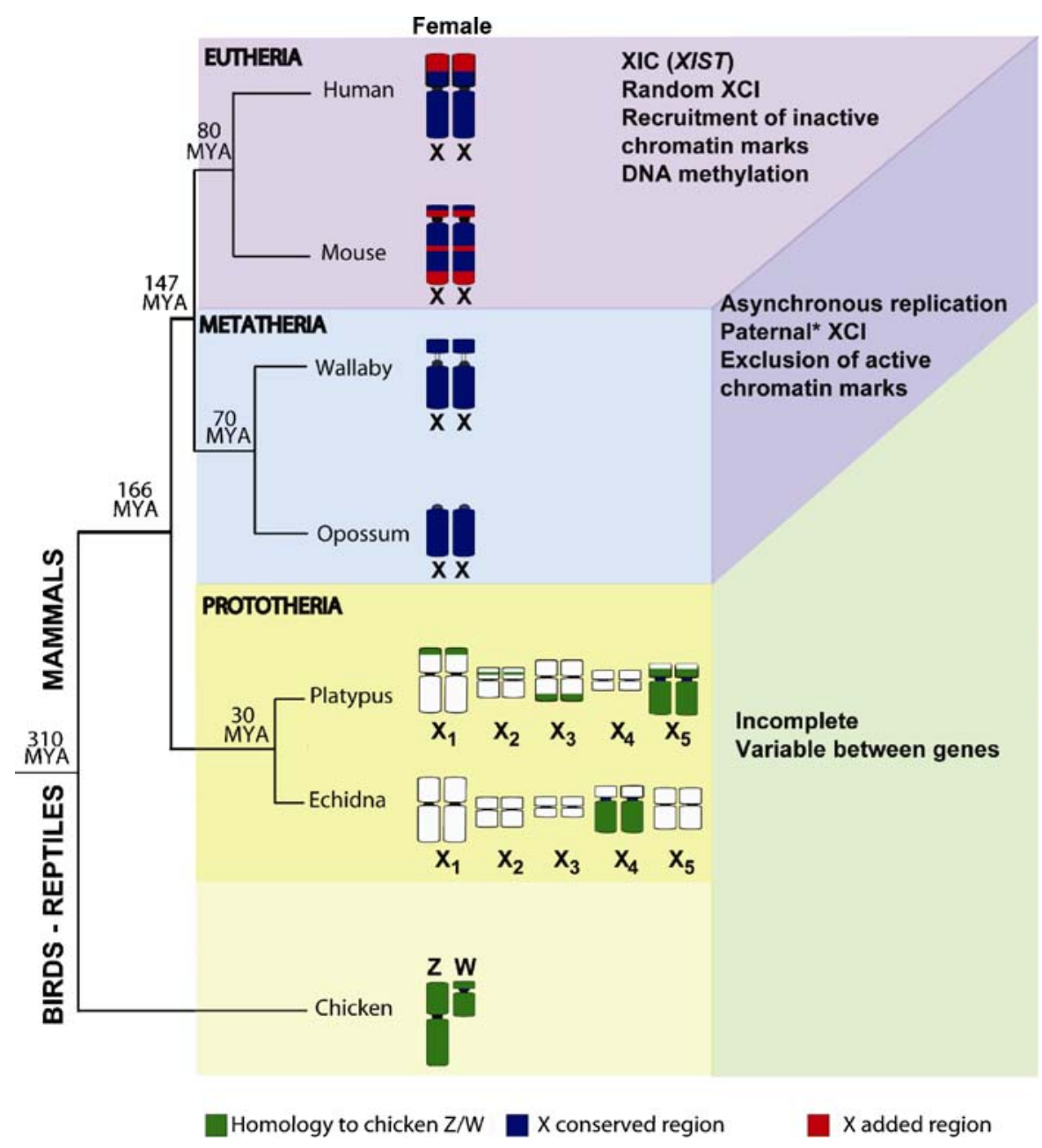

the paternal $\mathrm{X}$ was found always to be the late replicating one (Sharman 1971). Thus, marsupial X inactivation was the first example of parent-specific gene expression, ("genomic imprinting") to be reported in mammals (Cooper et al. 1971).

Despite its significance with respect to understanding $\mathrm{X}$ inactivation and genomic imprinting, paternalspecific silencing of the marsupial $X$ is poorly studied. Paternal $\mathrm{X}$ inactivation has been described for a mere four genes spread over nine species in which they were polymorphic (Table 1), and the lack of consistency and small numbers of genes has made it difficult to establish the rules of marsupial $\mathrm{X}$ inactivation. Older studies determined the inactivation status of the classic G6PD, PGK1, and GLA (with indirect evidence about HPRT1) using isozyme analysis (reviewed in Cooper et al. 1993). Inactivation was shown to be incomplete and locus-specific. For instance, $G 6 P D$ in D. virginiana was incompletely silenced in most tissues whereas PGK1 showed complete inactivation (Cooper et al. 1993). Confusingly, the patterns of inactivation varied between species, with the paternal $G 6 P D$ allele being completely silenced in somatic tissues from two kangaroo species (Macropus robustus and Macropus rufogriseus), yet was partially active in tissues from the North American opossum (Didelphis virginiana).

More sensitive molecular methods have been since used to detect transcription of two genes (G6PD and PGK1) on the marsupial X. SNuPE (Single Nucleotide Primer Extension) assay results confirmed isozyme studies showing complete silencing of the paternal copy of G6PD in somatic tissues in the wallaroo (Macropus robustus) (Watson et al. 2000), and allele-specific RT-PCR was used to show mostly complete inactivation of the paternal $G 6 P D$ 
Table 1 Genes and species used to study $\mathrm{X}$ inactivation in marsupials
Isozyme data reviewed in Cooper et al. 1993. ${ }^{1}$ Watson et al. 2000; ${ }^{2}$ Hornecker et al. 2007; ${ }^{3}$ Koina et al. 2005.

\begin{tabular}{|c|c|c|c|c|}
\hline \multirow[t]{2}{*}{ Gene } & \multirow[t]{2}{*}{ Species } & \multirow[t]{2}{*}{ Method } & \multicolumn{2}{|l|}{ Inactivation Status } \\
\hline & & & Somatic tissues & $\begin{array}{l}\text { Cultured } \\
\text { fibroblasts }\end{array}$ \\
\hline \multirow[t]{5}{*}{$G 6 P D$} & Macropus robustus & Isozyme & Complete & Incomplete \\
\hline & & $\mathrm{SNuPE}^{1}$ & Complete & Incomplete \\
\hline & Macropus rufogriseus & Isozyme & Complete & Incomplete \\
\hline & Didelphis virginiana & Isozyme & Incomplete & Incomplete \\
\hline & Monodelphis domestica & Allele-specific RT-PCR ${ }^{2}$ & Mostly complete & - \\
\hline \multirow[t]{2}{*}{$G L A$} & Antechinus stuarttii & Isozyme & Complete & Complete \\
\hline & Kangaroo hybrids & Isozyme & Complete & Complete \\
\hline \multirow[t]{2}{*}{$H P R T$} & Didelphis virginiana & Isozyme & - & Incomplete \\
\hline & Macropus & & - & Incomplete \\
\hline \multirow[t]{5}{*}{$P G K$} & Macropus giganteus & Isozyme & Tissue specific & Incomplete \\
\hline & Macropus parryi & Isozyme & Tissue specific & Incomplete \\
\hline & Trichosurus vulpecula & Isozyme & Tissue specific & - \\
\hline & Didelphis virginiana & Isozyme & Complete & Complete \\
\hline & Monodelphis domestica & $\mathrm{SNuPE}^{2}$ & Incomplete & - \\
\hline$S L C 16 A 2$ & Macropus eugenii & RNA-FISH ${ }^{3}$ & - & Incomplete \\
\hline
\end{tabular}

allele in the South American opossum (M.domestica) (Hornecker et al. 2007). However, allele-specific RTPCR revealed expression of paternal PGK1 in most tissues. These results were further verified with a SNuPE assay and hot-stop RT-PCR, which provides more quantitative data, demonstrating that the paternal PGK1 allele was often expressed at the same, or occasionally even greater, level as the maternal copy (Hornecker et al. 2007).

Thus, although the paternal $\mathrm{X}$ is usually preferentially inactivated in all marsupials, patterns of gene expression on the marsupial $\mathrm{X}$ may differ between loci, between tissues, and between species.

Marsupial inactivation at the cellular level

Partial inactivation of the paternal allele of $G 6 P D$, HPRT1 and PGK1 observed in fibroblasts of most species and some somatic tissues, could be the result of uniformly low expression from the paternal $\mathrm{X}$ in every cell, or to a mix of cells, some with both copies active and others with monoallelic expression (Deakin et al. 2008a). None of these molecular assays discriminate between the two explanations. An alternative method for detecting inactivation at the transcript level is RNA-FISH, a technique that detects the nascent transcript at the site of transcription within individual nuclei. RNA-FISH was used to determine the inactivation status of $S L C 16 A 2$ on the tammar wallaby $\mathrm{X}$ chromosome. In $76 \%$ of nuclei examined, expression from just one allele was observed, and in $17 \%$ both were transcribed (a further $6 \%$ had no signal and $1 \%$ with 3 signals per nucleus) (Koina et al. 2005). It remains to be determined if cells with monoallelic expression are all transcribing only the maternal copy of SLC16A2, but these data suggest that partial expression of genes on the inactive $\mathrm{X}$ is due to some cells expressing both alleles and others just one.

Obviously information on the activity of many more genes on the marsupial $\mathrm{X}$ is required to understand the extent of marsupial $\mathrm{X}$ inactivation, and the patterns of variation. Current efforts of our lab are focussed on creating an activity map of the $\mathrm{X}$ chromosomes of both the opossum and tammar wallaby. A comparison of these maps between the two species will help to delineate the common features of marsupial $\mathrm{X}$ inactivation.

Sex chromosome elimination in marsupials

Several members of the bandicoot family (Peramelidae) take $\mathrm{X}$ chromosome inactivation to an extreme, a fascinating but surprisingly under-examined variation 
on the paternal $\mathrm{X}$ inactivation observed in other marsupials. They eliminate one sex chromosome (one $\mathrm{X}$ in females and the $\mathrm{Y}$ in males) in somatic tissues at different stages of development (Hayman and Martin 1974). This elimination varies between tissues from loss of one sex chromosome in all cells to loss only in a subset of cells (Hayman and Martin 1965). A polymorphism in the PGK1 protein in Southern brown bandicoots (Isodon obesulus) has provided some evidence that it is always the paternal $\mathrm{X}$ that is eliminated in female somatic tissues (Johnston et al. 2002). In this species, as in other marsupials, the paternal $\mathrm{X}$ is late replicating, as is the $Y$ (Johnston et al. 2002). The mechanism of sex chromosome loss remains unknown, but the preferential elimination of the paternal $\mathrm{X}$ and its delayed replication suggest a connection with $\mathrm{X}$ inactivation.

Marsupial X inactivation is not controlled by XIST

One of the most striking differences between marsupial and eutherian XCI is the absence of an XIST gene from the marsupial $\mathrm{X}$ chromosome. XIST and other neighbouring non-coding RNAs within the $\mathrm{X}$ inactivation centre play critical roles in $\mathrm{X}$ inactivation in eutherians, including the counting of the number of $\mathrm{X}$ chromosomes, the choice of the $\mathrm{X}$ chromosome to be inactivated and the initiation of silencing.

After many years of searching failed to find an XIST orthologue in marsupials, careful assembly and sequencing of the regions that flank this gene in humans and mice showed that XIST does not exist. In fact, genes flanking the XIC in humans, map to two different regions of the $\mathrm{X}$ chromosome in both the opossum and tammar wallaby (Davidow et al. 2007; Hore et al. 2007; Shevchenko et al. 2007; Deakin et al. 2008b), indicating that this region has been significantly rearranged since the divergence of marsupials and eutherians. Comparisons with genes in the orthologous region in birds and frogs suggest that some regions of the non-coding RNAs from within the XIC (including XIST) were derived from protein-coding genes, some of which are present in marsupials, but all of which have succumbed to pseudogenisation in eutherians (Duret et al. 2006). Integration of mobile elements during marsupial/ eutherian divergence or early in the eutherian lineage may have augmented the function of the proto-XIST gene, allowing it to evolve a role in $\mathrm{X}$ inactivation
(Elisaphenko et al. 2008). Another of these noncoding RNAs is TSIX, the non-coding RNA with a role in counting and choice in mice but not humans, also appears to have evolved in early eutherians, with the participation of repeat elements (Cohen et al. 2007).

The absence of XIST and disruption of its flanking markers means that $\mathrm{X}$ inactivation in marsupials cannot be under the control of the same XIC as it is in eutherians. Is it possible that there is a marsupial specific inactivation centre? Early studies of gene order and expression suggested a polarity in expression (Graves and Dawson 1988). However, the substantial rearrangement of the $\mathrm{X}$ between opossum and wallaby suggests that, rather, marsupial $\mathrm{X}$ inactivation is achieved on a gene by gene basis (Deakin et al. 2008b). Perhaps the evolution of XIST has imposed a chromosome-wide control on the $\mathrm{X}$ in eutherians.

\section{Epigenetic marks involved in marsupial XCI}

The human inactive $\mathrm{X}$ is visible under the microscope as a densely staining sex chromatin or "Barr body". No Barr body was distinguishable in the nucleus of adult possum cells (McKay et al. 1987), although it was obvious in the early embryo of a dasyurid marsupial, Antechinus stuartii (Johnston and Robinson 1987).

The heterochromatic state of the eutherian inactive $\mathrm{X}$ chromosome is now known to be due to the specific pattern of chromatin marks that distinguish it from its active homologue (reviewed in Heard 2005). Histone modifications appear at the time when inactivation becomes irreversible, making them candidates for being part of the 'lock-in' system of the silent state (Kohlmaier et al. 2004). DNA methylation at 5'CpG dinucleotides which appears later, seems to be involved in the maintenance of the inactive state (Lock et al. 1987; Norris et al. 1991; Sado et al. 2004). Thus, it is of great interest to compare this profile of modifications in marsupials, and to explore their potential role in marsupial $\mathrm{X}$ chromosome inactivation.

The eutherian inactive $\mathrm{X}$ chromosome loses histone modifications associated with transcriptional activity (H3K4me2, H3K9ac, H4Kac) and accumulates marks associated with inactivity (H3K9me2 and H3K27me3) (Keohane et al. 1996; Heard et al. 2001; 
Chaumeil et al. 2002; Okamoto et al. 2004). These three active marks are also lost from the marsupial $\mathrm{X}$ (Wakefield et al. 1997; Koina et al. 2009). However, in metaphase spreads, no enrichment of repressive marks $\mathrm{H} 3 \mathrm{~K} 9 \mathrm{me} 2$ or $\mathrm{H} 3 \mathrm{~K} 27 \mathrm{me} 3$ could be detected on one of the two wallaby $\mathrm{X}$ chromosomes (Koina et al. 2009). Indeed, some of our preliminary results suggest that H3K27me3 is not stably enriched on the inactive $\mathrm{X}$ in interphase (Chaumeil et al. in preparation). As recruitment of $\mathrm{H} 3 \mathrm{~K} 27 \mathrm{me} 3$, and probably also of $\mathrm{H} 3 \mathrm{~K} 9 \mathrm{me} 2$, depends on XIST RNA localisation in eutherians (Kohlmaier et al. 2004), their absence on the marsupial inactive $\mathrm{X}$ chromosome is consistent with the absence of the XIST gene from the marsupial genome. These modifications are suggested to help stabilise the inactive state of the eutherian X (Silva et al. 2003), so their absence from the marsupial $\mathrm{X}$, at least at metaphase, might render $\mathrm{XCI}$ less stable in marsupials, as suggested by the observations of Kaslow and Migeon (1987).

In eutherians, $\mathrm{X}$ chromosome inactivation is accompanied by methylation of $5^{\prime}$ CpGs (Mohandas et al. 1981; Graves 1982) although this appears to stabilize, rather than initiate, inactivation (Keohane et al. 1998). In marsupials there seems not to be methylation differences between the active and inactive $\mathrm{X}$ chromosomes, although this conclusion has been based on the study of just two genes, G6PD and PGK1. Kaslow and Migeon (1987) failed to find methylation differences in the G6PD 5' CpGs of the American opossum (Didelphis virginiana), using methylation sensitive restriction enzymes. More recently, Hornecker et al (2007) used bisulfite sequencing to demonstrate that $5^{\prime}$ CpG islands of G6PD and PGK1 were not differentially methylated in $M$. domestica. The methylation status of the wallaroo G6PD 5' $\mathrm{CpG}$ island gene was also determined using bisulfite sequencing, but no differential methylation was detected (Loebel and Johnston 1996).

However, one study is inconsistent with the conclusion that there are no DNA methylation differences between the active and inactive $\mathrm{X}$ chromosomes in marsupials. A chromosome-wide examination using methylation sensitive restriction enzymes on metaphase chromosomes followed by in situ nick translation showed considerable methylation of the paternal X (Loebel and Johnston 1993). Similarly, it was thought that imprinting of $I G F 2$ in marsupials was independent of methylation (Weidman et al. 2004), but this has recently been shown to be untrue, with methylation differences demonstrated in a previously unidentified $5^{\prime}$ untranslated exon of the opossum IGF2 gene (Lawton et al. 2008). Obviously we must probe the methylation status of more genes before we can rule out a role for methylation in marsupial $\mathrm{X}$ inactivation.

Thus, these studies demonstrated that loss of active histone marks is a common feature of marsupial and eutherian $\mathrm{X}$ chromosome inactivation. On the contrary, it seems that the marsupial inactive $\mathrm{X}$ fails to recruit the same inactive marks as the eutherian inactive $X$, at least in a stable manner. This could be due to the lack of the XIST gene in marsupials that is involved in recruiting repressive marks for stabilisation of the silencing state, and could explain, at least partially, why $\mathrm{X}$ chromosome inactivation is less stable in marsupials than in eutherians.

\section{Meiotic sex chromosome inactivation (MSCI)}

In eutherian mammals, the $\mathrm{X}$ (and $\mathrm{Y}$ ) chromosome is inactivated during spermatogenesis, presumably to account for gene dosage imbalances between $\mathrm{X}$ - and Y- bearing sperm (Monesi 1965; Lifschytz and Lindsley 1972). MSCI has recently been discovered in opossum by analysing expression of nine X-borne housekeeping and three germcell-specific genes in meiotic pachytene spermatocyctes. Seven of these genes showed a decrease in transcription level during spermatogenesis, suggesting that MSCI does occur in marsupials (Hornecker et al. 2007). Namekawa et al (2007) showed that marsupial MSCI shares many common features with MSCI in eutherians, including the binding of heterochromatin-associated proteins (HP1 $\beta$ and HP1 $\gamma$ ) and histone variant $\gamma \mathrm{H} 2 \mathrm{AX}$. However, HP1 $\beta$ and HP1 $\gamma$ binding occurs earlier in the opossum than it does in the mouse, being detectable by early pachytene. MSCI in mouse is not controlled by Xist (McCarrey et al. 2002; Turner et al. 2002), but two proteins, BRCA1 and kinase ATR, appear to contribute to chromatin condensation (Turner et al. 2004). Expression of BRCA1 and ATR during opossum spermatogenesis is consistent with a role in MSCI (Hornecker et al. 2007). Following meiosis, silencing of the $\mathrm{X}$ chromosome persists into spermiogenesis and is correlated with HP $1 \beta$ and HP1 $\gamma$ and enrichment and H3-K9 trimethylation (Hornecker et al. 2007; Namekawa et al. 2007). 
It was proposed (Cooper 1971) that in marsupials, the paternal $\mathrm{X}$, as well as the $\mathrm{Y}$, is inactivated during male meiosis and simply stays inactive throughout fertilization and development. H3K9me3 and/or HP1 in MSCI may represent imprint marks that keep the paternal $\mathrm{X}$ inactive or allow subsequent inactivation of paternal $\mathrm{X}$ in the embryo. For example, H3K9me3 is also found enriched on the inactive $\mathrm{X}$ in interphase in somatic female cells (Chaumeil et al, in preparation).

$\mathrm{X}$ inactivation in an ancestral therian mammal

Is MSCI ancestral? Is imprinted $\mathrm{X}$ inactivation ancestral? After the demonstration of paternal $\mathrm{X}$ inactivation in marsupials, paternal $\mathrm{X}$ inactivation was observed in the extra-embryonic tissues of rodents (Takagi and Sasaki 1975; Wake et al. 1976; West et al. 1977). Paternal X inactivation was not found in humans (Zeng and Yankowitz 2003) but was recently demonstrated in cattle (Dindot et al. 2004), suggesting that it was ancestral in eutherians and lost in humans. It has been proposed that imprinted $\mathrm{X}$ inactivation is ancestral since it occurs in both marsupials and eutherians (Cooper et al. 1971, 1993). Supporting this hypothesis is the observation that imprinted inactivation in mouse extra-embryonic tissues, like marsupial $\mathrm{X}$ inactivation, is less stable and incomplete, and does not involve DNA methylation (Huynh and Lee 2005). However this imprinted $\mathrm{X}$ inactivation in mice relies on Xist, which cannot be the case in marsupials (Marahrens et al. 1997; Okamoto et al. 2005). In mice, imprinted inactivation of the paternal $\mathrm{X}$ occurs very early in development, and is stabilized in the extraembryonic tissues. However, the paternal $\mathrm{X}$ is reactivated in blastocysts prior to undergoing random $\mathrm{X}$ inactivation (Huynh and Lee 2003; Mak et al. 2004; Okamoto et al. 2005).

An extension of this hypothesis is that imprinted $\mathrm{X}$ inactivation is a carryover from MSCI, with the suggestion that the paternally derived $\mathrm{X}$ arrives in the embryo in a "pre-inactivated" state. This state persists in marsupials and the extraembryonic tissues of eutherians but has been lost in the somatic cells of eutherians with the more recent evolution of randomness (Huynh and Lee 2005). Demonstration that the paternal $\mathrm{X}$ arrives inactive in the embryo would be very important, for it would establish an evolutionary link between MSCI and X inactivation. However, in mice the paternal $\mathrm{X}$ arrives active in the zygote and initiation of its inactivation at the 4 cell stage is independent of its inactivation during meiosis (Okamoto et al. 2004, 2005; Patrat et al. 2009). A more likely explanation is that an imprint is deposited on the paternal $\mathrm{X}$ chromosome during MSCI that allows the subsequent inactivation of this chromosome during early development of marsupials and eutherians. Continued investigations of marsupial MSCI and XCI are required to test this hypothesis.

Future directions for marsupial dosage compensation studies

Many basic questions regarding $\mathrm{X}$ inactivation in marsupials remain unanswered. Does $\mathrm{X}$ inactivation in marsupials result in full or partial (or any?) dosage compensation between males and females? Is there dosage compensation between the $\mathrm{X}$ and autosomes as there is in eutherians? Is inactivation via transcriptional repression as it is in eutherians (Graves and Gartler 1986)? Is there any evidence of differential methylation? Is inactivation controlled across the $\mathrm{X}$ by a marsupial XIST-like gene, or does inactivation occur on a gene-by-gene basis? Armed with knowledge of the genic content and sequence of the marsupial $\mathrm{X}$ chromosome, we are able to work towards answering these questions.

\section{Monotremes}

Monotremes are a basal branch of mammals, a subclass (Prototheria) that diverged from the eutherian-marsupial lineage about 166 million years ago. They have a curious mix of reptilian and mammalian features, exemplified by feeding their young milk, like all other mammals, yet laying eggs similar to those of snakes. Monotremes are represented by four species of echidna and just one extant species of platypus that is limited to Australia. These animals are difficult to breed reliably in captivity (Temple-Smith and Grant 2001), making it troublesome to obtain tissue samples for dosage compensation studies, and virtually impossible to acquire monotreme embryos. However, advances are being made in monotreme husbandry which may improve our chances of obtaining such samples in the future. 
Despite the lack of a laboratory model monotreme, we have recently gained insight into dosage compensation for the platypus. This has followed the clarification, greatly assisted by the platypus genome project (Warren et al. 2008), of the genic content of the platypus sex chromosomes.

\section{Unique sex chromosome system}

The unique features of monotremes include bizarre sex chromosomes. Like other mammals, they have male heterogamety, but their sex chromosome system is rather complex. The platypus (Ornithorhynchus anatinus) has ten sex chromosomes; females have five pairs of $\mathrm{X}$ chromosomes $\left(\mathrm{X}_{1} \mathrm{X}_{2} \mathrm{X}_{3} \mathrm{X}_{4} \mathrm{X}_{5}\right)$ and males have five different $X\left(X_{1} X_{2} X_{3} X_{4} X_{5}\right)$ and five different $Y$ chromosomes $\left(\mathrm{Y}_{1} \mathrm{Y}_{2} \mathrm{Y}_{3} \mathrm{Y}_{4} \mathrm{Y}_{5}\right)$ (Grützner et al. 2004; Rens et al. 2004). In echidnas (Tachyglossus aculeatus), females have five pairs of $\mathrm{X}$ chromosomes and males have five $\mathrm{X}$ chromosomes $\left(\mathrm{X}_{1} \mathrm{X}_{2} \mathrm{X}_{3} \mathrm{X}_{4} \mathrm{X}_{5}\right)$ and four $Y$ chromosomes $\left(Y_{1} Y_{2} Y_{3} Y_{4}\right)$ (Rens et al. 2007). In both of these species, the $\mathrm{X}$ and $\mathrm{Ys}$ form a multivalent translocation chain during male meiosis, in which $\mathrm{X}$ and $\mathrm{Y}$ chromosomes pair within terminal pseudoautosomal regions to form a chain of alternating $\mathrm{X}$ and $\mathrm{Y}$ chromosomes, which segregate into $\mathrm{X}$ bearing (female-determining) and Y-bearing (maledetermining) sperm (Grützner et al. 2004).

The gene content of the $\mathrm{X}$ chromosomes was expected to be related to that of the therian $\mathrm{X}$. However, contrary to earlier reports, monotreme sex chromosomes share no homology with the those of therians (Veyrunes et al. 2008). Genes from the therian $\mathrm{X}$ chromosome, including those flanking XIST in eutherians, lie on chromosome 6 (Waters et al. 2005; Hore et al. 2007; Veyrunes et al. 2008). Intriguingly, monotreme $\mathrm{X}$ chromosomes share homology with the $\mathrm{Z}$ chromosome of birds (Fig. 1), and include the bird candidate sex determining gene DMRT1 (Rens et al. 2007; Veyrunes et al. 2008). The large platypus $X_{5}$ contains the largest region homologous to the chicken $Z$ (Veyrunes et al. 2008), with smaller areas of homology spread over $\mathrm{X}_{1}, \mathrm{X}_{2}$ and $\mathrm{X}_{3}$. Platypus $\mathrm{X}$ chromosomes also share some homology with chicken chromosomes 2, 3, 12, 13, 16 and 17.

Although the echidna has a similar multiple XY complex, the platypus and echidna chains are not entirely homologous. Chromosome painting has shown that platypus $\mathrm{X}_{4}$ corresponds to echidna chromosome
27 , whereas echidna $X_{5}$ shares homology with platypus chromosome 12 (Rens et al. 2007), suggesting that four elements of the chain were in place by the time platypus and echidnas diverged, but different members were added independently in the platypus and echidna lineages. The gene content of the echidna X chromosomes is not completely known, but the region containing DMRT1 and other genes on platypus $\mathrm{X}_{5}$ have been localised to echidna $X_{4}$ (Rens et al. 2007).

This unexpected homology between the platypus $\mathrm{XY}$ and the bird ZW chromosomes suggests that early mammals had a bird-like sex chromosome system that was usurped by the evolution of the SRY gene from $S O X 3$ on an autosome that became redefined as an XY pair (Graves 2008).

Variable dosage compensation in the platypus

The need for dosage compensation in monotremes would appear to be extreme, since the multiple $\mathrm{X}$ chromosomes of the complex account for approximately $15 \%$ of the haploid genome. They are mostly unpaired by the $\mathrm{Y}$ chromosomes, which are smaller (5\%) and largely heterochromatic. Thus at least $12 \%$ of the genome is present in twice the dose in females as in males.

The different origin of the monotreme and therian sex chromosomes might suggest an independent origin of dosage compensation in this lineage. Are platypus $\mathrm{X}$ chromosomes inactivated as in mouse and man? Or is there a partial system of dosage compensation as has been described for birds, or a unique monotreme dosage compensation system? The homology between monotreme and bird sex chromosomes suggests that dosage compensation in these two lineages may be expected to share some common features, except that gene dosage is in the opposite direction; in birds, it is 2:1 in favour of males, and in platypus 2:1 in favour of females, so how a common mechanism for dosage compensation could function is not obvious.

Early studies of replication timing of the large $\mathrm{X}_{1}$ in platypus and echidna fibroblasts found no asynchronous replication of the unpaired region of this chromosome (Murtagh 1977; Wrigley and Graves 1988). Although echidna lymphocytes showed asynchronous replication of the short arm of $\mathrm{X}_{1}$ (Wrigley and Graves 1988), this region pairs with $Y_{1}$ and would not require dosage compensation (Grützner et 
al. 2003). These inconsistent results did not make a compelling case for $\mathrm{X}$ inactivation.

Now that genes have been assigned to platypus $\mathrm{X}$ chromosomes, and considerable sequence is available, more sophisticated molecular studies can be performed. These show, surprisingly, that platypus dosage compensation is partial and differs between genes (Deakin et al. 2008a). Female to male expression ratios were determined for 19 genes located on platypus X chromosomes. Nine of these genes lie in pseudoautosomal regions. Expression ratios ranged from $0.7-1.2$, indicating that their Y homologues are active. Two pseudoautosomal genes had ratios around 2.0, suggesting that either their $\mathrm{Y}$ copies are not expressed or that the sequence of the $\mathrm{Y}$ homologues has diverged from that of the $\mathrm{X}$ copy. Of the 10 genes in X-specific regions, half had a female to male ratio close to one (complete compensation), two had an intermediate ratio of around 1.4 (partial compensation), and three showed no evidence of compensation, having a ratio close to two (Deakin et al. 2008a) (Table 2). Considerable variation between individuals was observed.

This range of levels of compensation between genes resembles the variable and incomplete dosage compensation observed in birds (Ellegren et al. 2007; Itoh et al. 2007). Microarray comparisons of male: female expression ratios in chicken and zebrafinch produced a distribution of genes, with some fully compensated, some uncompensated, but most some- where between (Itoh et al. 2007). The distribution of compensated and uncompensated genes along the $\mathrm{Z}$ chromosome was not random: a region in which most genes are not compensated ('peak') has been found on the long arm of the chicken $\mathrm{Z}$, and one region contains genes that are generally compensated ("valley'). Although Mank and Ellegren (2009) argue that these two regions are an artefact of the analysis approach, it is intriguing that this 'valley' of compensation lies near the $M H M$ (male hypermethylated) locus on chicken Zp (Melamed and Arnold 2007), which transcribes a non-coding RNA only in females which accumulates near the DMRT1 locus (Teranishi et al. 2001). With data for only ten X-specific genes in platypus, it is difficult to determine if a similar trend exists in this species. Intriguingly, two genes on $\mathrm{X}_{5}$ (DMRT2 and SLC1A1) found in the valley of compensation on the chicken $\mathrm{Z}$ appear not to be compensated in platypus, suggesting that XX females are more tolerant of higher expression of these genes than $\mathrm{ZZ}$ males.

As for marsupials, partial expression in monotremes could be due to a uniformly lower expression of one allele than the other in all cells, or to a mixture of cells in which one or both alleles is active. Examination of the probability of expression via RNA-FISH strongly favoured the latter explanation. Genes showed different frequencies of two X-active nuclei, ranging from $20 \%$ to $53 \%$, with an average of $45 \%$ of nuclei with biallelic expression. This suggests

Table 2 Female:male expression ratios and frequency of monoallelic expression for platypus X-specific genes (Deakin et al. 2008a)

\begin{tabular}{llll}
\hline Chromosome & Gene & Female:Male Ratio & Percent of nuclei with monoallelic expression \\
\hline Compensated & & & \\
$\mathrm{X}_{1}$ & Ox_plat_124086 & 1.10 & 46 \\
$\mathrm{X}_{5}$ & ZNF474,LOOX* & $1.01,1.06$ & 53 \\
$\mathrm{X}_{3}$ & APC & 1.17 & 48 \\
$\mathrm{X}_{5}$ & SHB & 1.23 & 53 \\
Partially Compensated & & & \\
$\mathrm{X}_{5}$ & FBXO10 & 1.37 & 50 \\
$\mathrm{X}_{5}$ & EN14997 & 1.40 & 61 \\
Not compensated & & & \\
$\mathrm{X}_{5}$ & SEMA6A & 1.82 & 74 \\
$\mathrm{X}_{5}$ & DMRT2 & 2.04 & 47 \\
$\mathrm{X}_{5}$ & SLC1A1 & 2.78 & 45 \\
\hline
\end{tabular}

*These genes were contained within a single BAC used as a probe for RNA-FISH 
some form of transcriptional silencing (Deakin et al. 2008a). Single Nucleotide Polymorphisms (SNPs) found in three of these X-specific genes were biallelically expressed, with equal expression of each allele, indicating that the monallelic expression detected is stochastic, and not imprinted as it is in marsupials (Deakin et al. 2008a). This stochastic expression may be co-ordinately regulated in platypus as two genes lying $500 \mathrm{~kb}$ apart always showed inactivation of the same X. Studying genes that are further apart will show if coordination is at a local level or is chromosome-wide.

Is stochastic monoallelic expression, such as that described for the platypus, a basic mechanism from which X inactivation arose? Ohlsson et al. (2001) proposed that $\mathrm{X}$ inactivation and genomic imprinting arose from stochastic expression that later became more strictly controlled. A recent study has shown that stochastic monoallelic expression is widespread on human autosomes, affecting perhaps as many as 1000 genes in the human genome (Gimelbrant et al. 2007). Most genes with stochastic expression also showed some level of biallelic expression, similar to that seen in the platypus. Further support for this hypothesis comes from experiments on mouse tetraploid cells, in which each $\mathrm{X}$ chromosome was found to have an independent probability of initiating $\mathrm{X}$ chromosome inactivation (Monkhorst et al. 2008). Interestingly, the "probability-promoting factor" is encoded outside of the Xist-Tsix-Xite region (Monkhorst et al. 2008) and the inactivation of a single $\mathrm{X}$ is locked in by a feedback mechanism, controlled by the $\mathrm{X}$ inactivation centre, that suppresses the inactivation of the active X (MlynarczykEvans et al. 2006). Incomplete platypus dosage compensation may result from the absence of such a feedback mechanism in a species with no XIST.

Future directions for monotreme dosage compensation studies

It will be important to extend these studies to many more platypus genes, potentially matching the microarray studies in birds. It would also be very advantageous to extend these studies to the echidna, which has many advantages as a monotreme model, especially if improved husbandry makes captive breeding more successful, and eggs available. To date, no molecular studies have been conducted on echidna dosage compensation. Echidna sex chromosomes share only four of the five XY elements of the chain so comparison of dosage compensation between platypus and echidna could provide information on whether differences in gene content direct dosage compensation.

Examining $\mathrm{X}$ inactivation in monotremes early in development could help answer many questions about the evolution of $X$ inactivation. Do monotremes have imprinted $\mathrm{X}$ inactivation early in development that is then lost, as in eutherians? Is dosage compensation more tightly controlled during development?

At this stage, it is unknown the extent (if any) to which chromatin modifications play a role in platypus dosage compensation. Hence, the logical next step is to examine these modifications in the platypus. Of particular interest would be those found in both marsupials and eutherians, such as the marks of the active $\mathrm{X}$.

\section{Concluding Remarks}

An understanding of dosage compensation in marsupials and monotremes will help us to unravel the evolutionary origins of dosage compensation in mammals. Our discovery that the $\mathrm{X}$ chromosomes in platypus have a bird-like partial dosage compensation system as well as homology to the bird $\mathrm{Z}$ chromosome, changes our picture of how and when $\mathrm{X}$ chromosome inactivation evolved in mammals (Fig. 1).

Our discovery that the therian XY chromosome pair is represented by an autosome in platypus, as well as in birds, dates the origin of the therian XY system to $145-166 \mathrm{MYA}$, comparatively recently. The ancestral mammal almost certainly had ZW or XY chromosomes with homology to the bird ZW chromosome pair (Graves 2008). Our discovery that dosage compensation in the platypus is only partial and locus specific, similar to birds, strongly suggests that monotremes have retained an ancestral compensation system.

The occurrence of $\mathrm{X}$ chromosome inactivation in marsupials as well as eutherians suggests that the marsupial and eutherian $\mathrm{X}$ inactivation systems share a common evolutionary origin. Observations that MSCI and paternal $\mathrm{X}$ inactivation occurs in marsupials and the extraembryonic tissues of eutherians, and is less stable, incomplete and lacking differential DNA methylation, suggests that the ancestral system involved paternal-specific 
inactivation, and ultimately evolved from MSCI (Huynh and Lee 2005).

However, the major differences in inactivation phenotype, and in the molecular mechanism of $\mathrm{X}$ inactivation in marsupials and eutherians, suggests an ongoing sophistication of $\mathrm{X}$ inactivation in eutherians. In particular the finding that imprinted XCI in mice depends on Xist (Marahrens et al. 1997; Okamoto et al. 2005), but is XIST-independent in marsupials (Duret et al. 2006; Davidow et al. 2007; Hore et al. 2007; Shevchenko et al. 2007) would require that an ancestral paternal, incomplete $\mathrm{X}$ inactivation mechanism was brought under chromosome-wide control by acquisition of the XIST locus early in eutherian evolution. Differences in the molecular mechanism of inactivation suggest that a more complete and stable system evolved with the addition of repressive histone marks and DNA methylation on the inactive X, which may be linked to the action of the non-coding XIST RNA.

Despite the differences in dosage compensation mechanisms between monotremes and therians, both systems may use similar molecular changes from an ancient molecular toolbox. As suggested by Ohlsson et al. (2001), X inactivation may have originally arisen from stochastic monoallelic expression that is now known for many genes on all chromosomes (Gimelbrant et al. 2007), and this hypothesis gains support from observations of the stochastic nature of the initiation of $\mathrm{X}$ inactivation in mice (Monkhorst et al. 2008). Our discovery of the stochastic expression of platypus $\mathrm{X}$ genes suggests that monotreme dosage compensation also represents a specialization of stochastic monoallelic expression.

This hypothesis proposes that alteration of chromatin structure (involving histone modification, DNA methylation, change in replication time) changes the probability of transcription of genes. Further characterisation of $\mathrm{X}$ inactivation in marsupials and monotremes will help to identify common elements of epigenetic control of gene action in mammals, and to determine how they were built into the complex system by which the eutherian $\mathrm{X}$ is inactivated.

\section{References}

Augui S, Filion GJ, Huart S et al (2007) Sensing X chromosome pairs before $\mathrm{X}$ inactivation via a novel $\mathrm{X}$ pairing region of the Xic. Science 318:1632-1636
Bacher CP, Guggiari M, Brors B et al (2006) Transient colocalization of $\mathrm{X}$-inactivation centres accompanies the initiation of X inactivation. Nat Cell Biol 8:293-299

Bininda-Emonds OR, Cardillo M, Jones KE et al (2007) The delayed rise of present-day mammals. Nature 446:507512

Brockdorff N, Ashworth A, Kay GF et al (1991) Conservation of position and exclusive expression of mouse Xist from the inactive X chromosome. Nature 351:329-331

Brown CJ, Lafreniere RG, Powers VE et al (1991) Localization of the $\mathrm{X}$ inactivation centre on the human $\mathrm{X}$ chromosome in Xq13. Nature 349:82-84

Carrel L, Willard HF (2005) X-inactivation profile reveals extensive variability in $\mathrm{X}$-linked gene expression in females. Nature 434:400-404

Carrel L, Park C, Tyekucheva S et al (2006) Genomic environment predicts expression patterns on the human inactive X chromosome. PLoS Genet 2:e151

Chaumeil J, Okamoto I, Guggiari M, Heard E (2002) Integrated kinetics of $\mathrm{X}$ chromosome inactivation in differentiating embryonic stem cells. Cytogenet Genome Res 99:75-84

Chaumeil J, Le Baccon P, Wutz A, Heard E (2006) A novel role for Xist RNA in the formation of a repressive nuclear compartment into which genes are recruited when silenced. Genes Dev 20:2223-2237

Cohen DE, Davidow LS, Erwin JA et al (2007) The DXPas34 repeat regulates random and imprinted $\mathrm{X}$ inactivation. Dev Cell 12:57-71

Cooper DW (1971) Directed genetic change model for X chromosome inactivation in eutherian mammals. Nature 230:292-294

Cooper DW, VandeBerg JL, Sharman GB, Poole WE (1971) Phosphoglycerate kinase polymorphism in kangaroos provides further evidence for paternal $\mathrm{X}$ inactivation. Nat New Biol 230:155-157

Cooper DW, Johnston PG, Graves JAM (1993) X-inactivation in marsupials and monotremes. Sem Dev Biol 4:117-128

Davidow LS, Breen M, Duke SE et al (2007) The search for a marsupial XIC reveals a break with vertebrate synteny. Chromosome Res 15:137-146

Deakin JE, Hore TA, Koina E, Graves JAM (2008a) The status of dosage compensation in the multiple $\mathrm{X}$ chromosomes of the platypus. PLoS Genet 4:e1000140

Deakin JE, Koina E, Waters PD et al (2008b) Physical map of two tammar wallaby chromosomes: a strategy for mapping in non-model mammals. Chromosome Res 16:1159-1175

Dindot SV, Kent KC, Evers B et al (2004) Conservation of genomic imprinting at the XIST, IGF2, and GTL2 loci in the bovine. Mamm Genome 15:966-974

Duret L, Chureau C, Samain S, Weissenbach J, Avner P (2006) The Xist RNA gene evolved in eutherians by pseudogenization of a protein-coding gene. Science 312:1653-1655

Elisaphenko EA, Kolesnikov NN, Shevchenko AI et al (2008) A dual origin of the Xist gene from a protein-coding gene and a set of transposable elements. PLoS ONE 3:e2521

Ellegren H, Hultin-Rosenberg L, Brunstrom B et al (2007) Faced with inequality: chicken do not have a general dosage compensation of sex-linked genes. BMC Biol 5:40

Gimelbrant A, Hutchinson JN, Thompson BR, Chess A (2007) Widespread monoallelic expression on human autosomes. Science 318:1136-1140 
Graves JAM (1967) DNA synthesis in chromosomes of cultured leucocytes from two marsupial species. Exp Cell Res 46:37-57

Graves JAM (1982) 5-azacytidine-induced re-expression of alleles on the inactive $\mathrm{X}$ chromosome in a hybrid mouse cell line. Exp Cell Res 141:99-105

Graves JAM (1995) The evolution of mammalian sex chromosomes and the origin of sex determining genes. Philos Trans R Soc Lond B Biol Sci 350:305-311 discussion 311-2

Graves JAM (2008) Weird animal genomes and the evolution of vertebrate sex and sex chromosomes. Annu Rev Genet 42:565-586

Graves JAM, Dawson GW (1988) The relationship between position and expression of genes on the kangaroo $\mathrm{X}$ chromosome suggests a tissue-specific spread of inactivation from a single control site. Genet Res 51:103-109

Graves JAM, Gartler SM (1986) Mammalian X chromosome inactivation: testing the hypothesis of transcriptional control. Somat Cell Mol Genet 12:275-280

Graves JAM, Koina E, Sankovic N (2006) How the gene content of human sex chromosomes evolved. Curr Opin Genet Dev 16:219-224

Grützner F, Deakin J, Rens W, El-Mogharbel N, Graves JAM (2003) The monotreme genome: a patchwork of reptile, mammal and unique features? Comp Biochem Physiol A Mol Integr Physiol 136:867-881

Grützner F, Rens W, Tsend-Ayush E et al (2004) In the platypus a meiotic chain of ten sex chromosomes shares genes with the bird Z and mammal X chromosomes. Nature 432:913-917

Hayman DL, Martin PG (1965) Sex chromosome mosaicism in the marsupial genera Isoodon and Perameles. Genetics 52:1201-1206

Hayman DL, Martin PG (1974) Mammalia I: Monotremata and Marsupialia. In: John B (ed) Animal Cytogenetics 4: Chordata. Gebruder Borntraeger, Berlin/Stuttgart, pp 1-110

Heard E (2005) Delving into the diversity of facultative heterochromatin: the epigenetics of the inactive X chromosome. Curr Opin Genet Dev 15:482-489

Heard E, Rougeulle C, Arnaud D et al (2001) Methylation of histone H3 at Lys-9 is an early mark on the X chromosome during $\mathrm{X}$ inactivation. Cell 107:727-738

Hore TA, Koina E, Graves JAM (2007) The region homologous to the X-chromosome inactivation centre has been disrupted in marsupial and monotreme mammals. Chromosome Res 15:147-161

Hornecker JL, Samollow PB, Robinson ES, Vandeberg JL, McCarrey JR (2007) Meiotic sex chromosome inactivation in the marsupial Monodelphis domestica. Genesis 45:696708

Human Genome Sequencing Center at Baylor College of Medicine (2009) Internet references. Retrieved from http://www.hgsc.bcm.tmc.edu/projects/wallaby 28/2/2009.

Huynh KD, Lee JT (2003) Inheritance of a pre-inactivated paternal $\mathrm{X}$ chromosome in early mouse embryos. Nature 426:857-862

Huynh KD, Lee JT (2005) X-chromosome inactivation: a hypothesis linking ontogeny and phylogeny. Nat Rev Genet 6:410-418

Itoh Y, Melamed E, Yang X et al (2007) Dosage compensation is less effective in birds than in mammals. J Biol 6:2
Johnston PG, Robinson ES (1987) X chromosome inactivation in female embryos of a marsupial mouse (Antechinus stuartii). Chromosoma 95:419-423

Johnston PG, Watson CM, Adams M, Paull DJ (2002) Sex chromosome elimination, $\mathrm{X}$ chromosome inactivation and reactivation in the southern brown bandicoot Isoodon obesulus (Marsupialia: Peramelidae). Cytogenet Genome Res 99:119-124

Johnston CM, Lovell FL, Leongamornlert DA et al (2008) Largescale population study of human cell lines indicates that dosage compensation is virtually complete. PLoS Genet 4:e9

Kaslow DC, Migeon BR (1987) DNA methylation stabilizes X chromosome inactivation in eutherians but not in marsupials: evidence for multistep maintenance of mammalian X dosage compensation. Proc Natl Acad Sci U S A 84:6210-6214

Keohane AM, O'Neill LP, Belyaev ND, Lavender JS, Turner BM (1996) X-Inactivation and histone $\mathrm{H} 4$ acetylation in embryonic stem cells. Dev Biol 180:618-630

Keohane AM, Lavender JS, O'Neill LP, Turner BM (1998) Histone acetylation and X inactivation. Dev Genet 22:65-73

Kirsch JAW, Lapointe FJ, Springer MS (1997) DNAhybridisation studies of marsupials and their implications for metatherian classification. Australian Journal of Zoology 45:211-280

Kohlmaier A, Savarese F, Lachner M et al (2004) A chromosomal memory triggered by Xist regulates histone methylation in X inactivation. PLoS Biol 2:E171

Koina E, Wakefield MJ, Walcher C et al (2005) Isolation, X location and activity of the marsupial homologue of SLC16A2, an XIST-flanking gene in eutherian mammals. Chromosome Res 13:687-698

Koina E, Chaumeil J, Greaves IK, Tremethick DJ, Graves JAM (2009) Specific patterns of histone marks accompany X chromosome inactivation in a marsupial. Chromosome Res

Lawton BR, Carone BR, Obergfell CJ et al (2008) Genomic imprinting of IGF2 in marsupials is methylation dependent. BMC Genomics 9:205

Lifschytz E, Lindsley DL (1972) The role of X-chromosome inactivation during spermatogenesis (Drosophila-allocycly-chromosome evolution-male sterility-dosage compensation). Proc Natl Acad Sci U S A 69:182-186

Lock LF, Takagi N, Martin GR (1987) Methylation of the Hprt gene on the inactive $\mathrm{X}$ occurs after chromosome inactivation. Cell 48:39-46

Loebel DA, Johnston PG (1993) Analysis of DNase 1 sensitivity and methylation of active and inactive $\mathrm{X}$ chromosomes of kangaroos (Macropus robustus) by in situ nick translation. Chromosoma 102:81-87

Loebel DA, Johnston PG (1996) Methylation analysis of a marsupial $\mathrm{X}$-linked $\mathrm{CpG}$ island by bisulfite genomic sequencing. Genome Res 6:114-123

Lyon MF (1961) Gene action in the X-chromosome of the mouse (Mus musculus L.). Nature 190:372-373

Lyon MF (1998) X-chromosome inactivation: a repeat hypothesis. Cytogenet Cell Genet 80:133-137

Mak W, Nesterova TB, de Napoles M et al (2004) Reactivation of the paternal $\mathrm{X}$ chromosome in early mouse embryos. Science 303:666-669

Mank JE, Ellegren H (2009) All dosage compensation is local: Gene-by-gene regulation of sex-biased expression on the chicken Z chromosome. Heredity 102:312-320 
Marahrens Y, Panning B, Dausman J, Strauss W, Jaenisch R (1997) Xist-deficient mice are defective in dosage compensation but not spermatogenesis. Genes Dev 11:156166

McCarrey JR, Watson C, Atencio J et al (2002) X-chromosome inactivation during spermatogenesis is regulated by an $\mathrm{Xist} / \mathrm{Tsix}$-independent mechanism in the mouse. Genesis 34:257-266

McKay LM, Wrigley JM, Graves JA (1987) Evolution of mammalian X-chromosome inactivation: sex chromatin in monotremes and marsupials. Aust J Biol Sci 40:397-404

Melamed E, Arnold AP (2007) Regional differences in dosage compensation on the chicken $\mathrm{Z}$ chromosome. Genome Biol 8:R202

Mikkelsen TS, Wakefield MJ, Aken B et al (2007) Genome of the marsupial Monodelphis domestica reveals innovation in non-coding sequences. Nature 447:167-177

Mlynarczyk-Evans S, Royce-Tolland M, Alexander MK et al (2006) X chromosomes alternate between two states prior to random X-inactivation. PLoS Biol 4:e159

Mohandas T, Sparkes RS, Shapiro LJ (1981) Reactivation of an inactive human $\mathrm{X}$ chromosome: evidence for $\mathrm{X}$ inactivation by DNA methylation. Science 211:393-396

Monesi V (1965) Differential rate of ribonucleic acid synthesis in the autosomes and sex chromosomes during male meiosis in the mouse. Chromosoma 17:11-21

Monkhorst K, Jonkers I, Rentmeester E, Grosveld F, Gribnau J (2008) X inactivation counting and choice is a stochastic process: evidence for involvement of an X-linked activator. Cell 132:410-421

Murtagh CE (1977) A unique cytogenetic system in monotremes. Chromosoma 65:37-57

Namekawa SH, VandeBerg JL, McCarrey JR, Lee JT (2007) Sex chromosome silencing in the marsupial male germ line. Proc Natl Acad Sci U S A 104:9730-9735

Norris DP, Brockdorff N, Rastan S (1991) Methylation status of $\mathrm{CpG}$-rich islands on active and inactive mouse $\mathrm{X}$ chromosomes. Mamm Genome 1:78-83

Ohlsson R, Paldi A, Graves JAM (2001) Did genomic imprinting and $\mathrm{X}$ chromosome inactivation arise from stochastic expression? Trends Genet 17:136-141

Ohno S (1967) Sex Chromosomes and Sex Linked Genes. Springer, Berlin

Okamoto I, Otte AP, Allis CD, Reinberg D, Heard E (2004) Epigenetic dynamics of imprinted $\mathrm{X}$ inactivation during early mouse development. Science 303:644-649

Okamoto I, Arnaud D, Le Baccon P et al (2005) Evidence for de novo imprinted X-chromosome inactivation independent of meiotic inactivation in mice. Nature 438:369-373

Patrat C, Okamoto I, Diabangouaya P et al. (2009) Dynamic changes in paternal X-chromosome activity during imprinted X-chromosome inactivation in mice. Proc Natl Acad Sci U S A

Rens W, Grutzner F, O'Brien PC et al (2004) Resolution and evolution of the duck-billed platypus karyotype with an X1Y1X2Y2X3Y3X4Y4X5Y5 male sex chromosome constitution. Proc Natl Acad Sci U S A 101:16257-16261

Rens W, O'Brien PC, Grützner F et al (2007) The multiple sex chromosomes of platypus and echidna are not completely identical and several share homology with the avian Z. Genome Biol 8:R243
Richardson BJ, Czuppon AB, Sharman GB (1971) Inheritance of glucose-6-phosphate dehydrogenase variation in kangaroos. Nat New Biol 230:154-155

Sado T, Okano M, Li E, Sasaki H (2004) De novo DNA methylation is dispensable for the initiation and propagation of $\mathrm{X}$ chromosome inactivation. Development 131:975-982

Sharman GB (1971) Late DNA replication in the paternally derived $\mathrm{X}$ chromosome of female kangaroos. Nature 230:231-232

Shevchenko AI, Zakharova IS, Elisaphenko EA et al (2007) Genes flanking Xist in mouse and human are separated on the $\mathrm{X}$ chromosome in American marsupials. Chromosome Res 15:127-136

Silva J, Mak W, Zvetkova I et al (2003) Establishment of histone h3 methylation on the inactive $\mathrm{X}$ chromosome requires transient recruitment of Eed-Enx1 polycomb group complexes. Dev Cell 4:481-495

Takagi N, Sasaki M (1975) Preferential inactivation of the paternally derived $\mathrm{X}$ chromosome in the extraembryonic membranes of the mouse. Nature 256:640-642

Temple-Smith P, Grant T (2001) Uncertain breeding: a short history of reproduction in monotremes. Reprod Fertil Dev 13:487-497

Teranishi M, Shimada Y, Hori T et al (2001) Transcripts of the MHM region on the chicken $\mathrm{Z}$ chromosome accumulate as non-coding RNA in the nucleus of female cells adjacent to the DMRT1 locus. Chromosome Res 9:147-165

Turner JM, Mahadevaiah SK, Elliott DJ et al (2002) Meiotic sex chromosome inactivation in male mice with targeted disruptions of Xist. J Cell Sci 115:4097-4105

Turner JM, Aprelikova O, Xu X et al (2004) BRCA1, histone $\mathrm{H} 2 \mathrm{AX}$ phosphorylation, and male meiotic sex chromosome inactivation. Curr Biol 14:2135-2142

Veyrunes F, Waters PD, Miethke P et al (2008) Bird-like sex chromosomes of platypus imply recent origin of mammal sex chromosomes. Genome Res 18:965-973

Wake N, Takagi N, Sasaki M (1976) Non-random inactivation of X chromosome in the rat yolk sac. Nature 262:580-581

Wakefield MJ, Keohane AM, Turner BM, Graves JAM (1997) Histone underacetylation is an ancient component of mammalian X chromosome inactivation. Proc Natl Acad Sci U S A 94:9665-9668

Warren WC, Hillier LW, Graves JAM et al (2008) Genome analysis of the platypus reveals unique signatures of evolution. Nature 453:175-183

Waters PD, Delbridge ML, Deakin JE et al (2005) Autosomal location of genes from the conserved mammalian $\mathrm{X}$ in the platypus (Ornithorhynchus anatinus): implications for mammalian sex chromosome evolution. Chromosome Res 13:401-410

Watson D, Jacombs AS, Loebel DA, Robinson ES, Johnston PG (2000) Single nucleotide primer extension (SNuPE) analysis of the G6PD gene in somatic cells and oocytes of a kangaroo (Macropus robustus). Genet Res 75:269-274

Weidman JR, Murphy SK, Nolan CM, Dietrich FS, Jirtle RL (2004) Phylogenetic footprint analysis of IGF2 in extant mammals. Genome Res 14:1726-1732

West JD, Frels WI, Chapman VM, Papaioannou VE (1977) Preferential expression of the maternally derived $\mathrm{X}$ chromosome in the mouse yolk sac. Cell 12:873-882 
Wrigley JM, Graves JAM (1988) Sex chromosome homology and incomplete, tissue-specific X-inactivation suggest that monotremes represent an intermediate stage of mammalian sex chromosome evolution. J Hered 79:115-118

Xu N, Tsai CL, Lee JT (2006) Transient homologous chromosome pairing marks the onset of $\mathrm{X}$ inactivation. Science 311:1149-1152
Zeng SM, Yankowitz J (2003) X-inactivation patterns in human embryonic and extra-embryonic tissues. Placenta 24:270 275

Zenger KR, McKenzie LM, Cooper DW (2002) The first comprehensive genetic linkage map of a marsupial: the tammar wallaby (Macropus eugenii). Genetics 162:321330 